\title{
Potential influence of phytochemicals from beetroot juice on postprandial glycaemia
}

\author{
P. C. Wootton-Beard and L. Ryan \\ ${ }^{1}$ Functional Food Centre, Oxford Brookes University, Gipsy Lane, Oxford, OX30BP, UK
}

Disturbances in the postprandial response to glucose and insulin sensitivity are now known to be critical for good health. In the case of Type 2 diabetes mellitus (T2DM), a number of compounds have been suggested to either reduce postprandial glycaemia or to affect the postprandial insulin response ${ }^{(1)}$. Polyphenols including flavanoids, phenolic acids, proanthocyanidins and tannins have been suggested to be able to modify glucose metabolism.

Beetroot juice has received attention in the scientific literature recently due to its nitrate content ${ }^{(2)}$. Beetroot also contains a number of bioactive compounds including phenolic acids and betalains as well as a high total antioxidant capacity (TAC) and total polyphenol (TP) content $^{(3)}$. The aim of this study was to elucidate the postprandial glycaemic profile following consumption of 50g carbohydrate $(\mathrm{CHO})$ from either a beetroot juice meal compared to a control meal matched for $\mathrm{CHO}$ composition or a positive control meal of white bread. After obtaining approval from Oxford Brookes University ethics committee, 17 healthy subjects consumed each meal in a randomised crossover design and measurements of blood glucose and blood insulin were collected over a 2.5 hour postprandial period. Results were analysed using repeated measures ANOVA followed by bonferroni correction.

\begin{tabular}{|c|c|c|c|c|c|c|c|c|}
\hline \multirow[t]{2}{*}{ Age (yrs) } & \multirow[t]{2}{*}{ BMI } & \multicolumn{4}{|c|}{ iAUC glucose $(\mathrm{mmol} / \mathrm{L})$} & \multicolumn{2}{|c|}{ iAUC insulin $(\mu \mathrm{IU} / \mathrm{mL})$} & \multirow[t]{2}{*}{ HPPI (mg/day) } \\
\hline & & BEET & MCON & $\mathrm{CON}$ & BEET & MCON & $\mathrm{CON}$ & \\
\hline $26.5 \pm 4.2$ & $23.5 \pm 2.6$ & $66.71 \pm 11.54$ & $78.07 \pm 14.77$ & $125.25 \pm 17.81$ & $1644.00 \pm 214.31$ & $1936.48 \pm 281.82$ & $1767.82 \pm 247.53$ & $1633.0 \pm 533.4$ \\
\hline
\end{tabular}

Data are displayed as mean \pm SE $(n=17)$. HPPI = habitual polyphenol intake. BMI $=$ weight $(\mathrm{kg}) /$ height $\left(\mathrm{m}^{2}\right)$.

Incremental area under the curve (iAUC) analysis revealed no significant differences between the beetroot juice (BEET) and the matched control $(\mathrm{MCON})$, both were significantly lower than the positive control $(\mathrm{CON})(P<0.05)$ for glucose and insulin using repeated measures ANOVA. Individual time points were compared and a significantly lower insulin response was detected at 15 minutes for BEET compared to MCON $(P<0.05)$. A lower glucose value was also detected at this time point although it did not reach significance $(P=0.06)$. We hypothesised that phytochemicals within beetroot juice may be responsible for reducing glucose uptake, however the $70 \mathrm{~mL}$ of beetroot juice given in this study may not have been sufficient to clearly observe this effect, further study is currently underway utilising a larger amount of beetroot juice compared to controls which are all in beverage form.

1. Hanhineva K, Torronen R, Bondia-Pons I et al. (2010) Int J Mol Sci 11, 1365-1402.

2. Webb AJ, Patel N, Loukogeorgakis S et al. (2008) Hypertension 51, 784-790.

3. Wootton-Beard PC, Moran A \& Ryan L (2011) Food Res Int 44, 217-224. 